\title{
Medidas de desempenho e eficiência, características da carcaça mensuradas por ultrassonografia e o consumo alimentar residual de ovinos ${ }^{1}$
}

\author{
Performance and efficiency measurements, carcass characteristics \\ obtained by ultrasound and the residual feed intake of sheep
}

Edson Ferraz Evaristo de Paula[a], Alda Lúcia Gomes Monteiro ${ }^{[b]}$, Odilei Rogério Prado ${ }^{[c]}$,Tiago Rafael Cosmo ${ }^{[d]}$, Nelson Santos Teixeira Junior ${ }^{[e]}$, Carlos Henrique Kulik ${ }^{[[]}$, Rodrigo de Almeida Teixeira[g]

\footnotetext{
[a] Zootecnista, mestre em Ciências Veterinárias, Universidade Federal do Paraná (UFPR), Curitiba, PR - Brasil, e-mail: efevaristo@globo.com

[b] Engenheira-agrônoma, doutora, professora do Departamento de Zootecnia, Universidade Federal do Paraná (UFPR), bolsista Produtividade em Pesquisa, CNPq, Curitiba, PR - Brasil, e-mail: aldaufpr@gmail.com

[c] Médico-veterinário, mestre, doutorando do Programa de Pós-Graduação em Ciências Veterinárias, Universidade Federal do Paraná (UFPR), Curitiba, PR - Brasil, e-mail: orpradovet@gmail com

[d] Graduando em Zootecnia, Universidade Federal do Paraná (UFPR), Curitiba, PR - Brasil, e-mail: trcosmo@gmail.com

[e] Engenheiro-agrônomo, mestrando em Agronomia, Universidade Federal do Paraná(UFPR),Curitiba,PR-Brasil, e-mail: saintreims@hotmail.com

[f] Graduando em Zootecnia, Universidade Federal do Paraná (UFPR), Curitiba, PR - Brasil, e-mail: henriqueckulik81@yahoo.com.br

[g] Zootecnista, doutor, professor do Departamento de Zootecnia, Universidade Federal do Paraná (UFPR), Curitiba, PR - Brasil, e-mail: rodrigoteixeira@pq.cnpq.br
}

\section{Resumo}

Avaliou-se o consumo alimentar residual (CAR) de 13 cordeiros visando o estímulo das correlações entre essa variável com medidas de desempenho e eficiência e características da carcaça e a verificação de diferenças em animais classificados pelo CAR (alto ou baixo). Após 25 dias de adaptação, os ovinos permaneceram confinados por 70 dias para mensuração do consumo de matéria seca (CMS) e foram pesados a cada 14 dias para obtenção do ganho de peso diário (GPD). Foram considerados o peso vivo inicial (PVI), peso vivo final (PVF), peso metabólico (PM), GPD, conversão alimentar (CA), eficiência alimentar (EA), CMS e CMS em percentual do PV (CMS\%PV). As avaliações de carcaça foram realizadas por ultrassom (frequência $6 \mathrm{MHz}$ ). 0 CAR se mostrou correlacionado $(\mathrm{p}<0,05)$ com o CMS $(+0,60)$ e com o CMS\%PV $(+0,71)$. Os cordeiros de CAR baixo tiveram menor CMS\%PV $(p=0,01)$ e tendência a apresentar menor CMS $(p=0,08)$. Não houve diferenças entre as classes de CAR para as variáveis GPD, PVI, PVF e PM, e os animais mais eficientes pelo CAR foram também mais eficientes pela CA e pela EA. A CA apresentou correlação $(p<0,05)$ com a EA $(-0,99)$, com o GPD $(-0,83)$ e com o PVF $(-0,61)$. Ao contrário da CA, o CAR apresentou independência fenotípica com medidas de crescimento e tamanho corporal e também não apresentou correlações ( $p>0,05)$ com as variáveis de carcaça estudadas. Confirmou-se o potencial do CAR como medida de eficiência alimentar para ovinos jovens em confinamento, sem prejuízos ao desempenho e ao acabamento das carcaças.

Palavras-chave: Área de olho de lombo. Conversão alimentar. Cordeiro. Deposição de gordura. Ultrassonografia.

$\overline{{ }^{1} \text { Prova de ganho de peso, LAPOC/GAMA, UFPR. }}$ 


\section{Abstract}

It was evaluated the residual feed intake (RFI) of 13 lambs in order to estimate the correlations between this variable with performance and efficiency and with carcass characteristics, as well to verify the differences among animals classified by the RFI (high or low). After 25 days of adaptation, the sheep were kept housed during 70 days for the measurement of dry matter intake (DMI) and were weighed every 14 days to obtain the average daily gain $(A D G)$. The experiments considered the initial live weight (ILW), final live weight (FLW), metabolic weight (MW), ADG, feed conversion rate (FCR), feed efficiency (FE), DMI, DMI in live weight percentual (DMI\%LW). Carcass assessments were made by ultrassonography (frequency $6 \mathrm{MHz}$ ). The RFI showed correlation $(p<0.05)$ with DMI $(+0.60)$ and with DMI\%LW $(+0.71)$. The low RFI lambs had low DMI\%LW $(p=0.01)$ and a trend to show lower DMI values ( $p=0.08)$. There were no differences among RFI classes to the ADG, ILW, FLW and MW, and the animals with better RFI efficiency also presented higher FCR and FE rates. The FCR was correlated ( $p<0.05)$ with FE (-0.99), ADG (-0.83) and FBW (-0.61). Unlike FCR, the RFI showed phenotipic independence with growth and body size traits and was not correlated with carcass measurements. This study confirmed the potential of RFI as a feed efficiency measurement for confined lambs, without impacting animal performance and carcass finishing.

Keywords: Rib eye area. Feed convertion rate. Lamb. Fat deposition. Ultrassonography.

\section{Introdução}

Diante do reconhecido desenvolvimento da cadeia produtiva da ovinocultura nos últimos anos, com aumento no consumo de carne ovina e com incremento nos impactos financeiros e sociais dessa cultura para a pecuária nacional, é importante atentar à necessidade de otimização da produção para atender a demanda e para tornar a ovinocultura mais competitiva. Uma alternativa para alcançar esse objetivo é a identificação e seleção de animais geneticamente superiores para características de relevância econômica.

A eficiência alimentar (EA) pode ser interpretada como a relação entre o ganho de peso e a quantidade de alimento ingerido, sendo o inverso denominado conversão alimentar (CA). Todavia a mensuração feita de tal maneira não leva em consideração as exigências de manutenção e crescimento dos indivíduos, resultando em medidas brutas de eficiência (ALMEIDA et al., 2004). Nesse contexto, o consumo alimentar residual (CAR) foi inicialmente sugerido para bovinos de corte como alternativa para determinação da eficiência alimentar (KOCH et al., 1963), e tem sido, recentemente, objeto de pesquisas também com a espécie ovina (KNOTT et al., 2010; REDDEN et al., 2011).
O CAR é uma importante ferramenta para identificação de animais que aproveitam mais eficientemente, em prol do ganho de peso, o alimento que lhes é oferecido. É definido como a diferença entre o consumo de matéria seca observado (CMSobs) e o consumo estimado (CMSest) para determinado nível de produção (KOCH et al., 1963), ou seja, quanto mais negativo o CAR, mais eficiente o animal. No entanto, observam-se, na literatura, trabalhos descrevendo que animais mais eficientes pelo CAR tendem a apresentar carcaças mais magras, com menor acabamento e menor gordura intramuscular (LANNA; ALMEIDA, 2004).

Desse modo, objetivou-se verificar as relações entre o CAR e outras medidas de desempenho e eficiência animal e avaliações realizadas na carcaça por ultrassonografia em ovinos da raça Dorper, bem como verificar diferenças relacionadas a essas características em animais classificados com base no CAR (alto ou baixo).

\section{Material e métodos}

O experimento foi realizado entre os meses de agosto de 2010 e novembro de 2010, no Laboratório de Produção e Pesquisa em Ovinos e Caprinos da Universidade Federal do Paraná (LAPOC-UFPR), 
situado na fazenda experimental do Canguiri, em Pinhais (PR), na região metropolitana de Curitiba.

Foram utilizados 13 cordeiros, candidatos a reprodutores, todos machos inteiros da raça Dorper, com $190 \pm 28$ dias de idade e 49,4 $\pm 4,3 \mathrm{~kg}$ de peso vivo (PV) inicial. Após 25 dias de adaptação ao manejo e à alimentação, os animais permaneceram confinados durante 70 dias em baias individuais com aproximadamente $1,0 \mathrm{~m} \mathrm{x} \mathrm{2,2} \mathrm{m} \mathrm{contendo} \mathrm{co-}$ chos individuais e bebedouros, para avaliação do consumo individual de alimento.

A alimentação foi ofertada ad libitum três vezes ao dia (às $8 \mathrm{~h}, 13 \mathrm{~h}$ e $17 \mathrm{~h}$ ), na forma de ração total misturada (Quadro 1), contendo feno de azevém picado e ração concentrada (proporção 40:60).

Quadro 1 - Composição da ração total misturada fornecida aos cordeiros

\begin{tabular}{lc}
\hline \multicolumn{1}{c}{ Ingredientes } & Inclusão (g/kg) \\
\hline Feno moído de azevém & 400,000 \\
Calcário calcítico & 10,199 \\
Casquinha de soja & 120,200 \\
Farelo de soja 44\% & 100,000 \\
Milho grão moído & 349,800 \\
Mistura mineral & \\
Monensina sódica & 19,780 \\
\hline
\end{tabular}

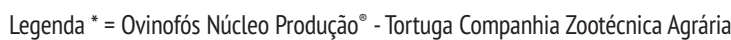
Fonte: Dados da pesquisa.

Nota: Níveis de garantia (por kg do produto) - P:61 g; Ca:267 g;S:35 g; Co:20 mg; Mn:2000 mg;Cu:350 mg;F: 610 mg;Se:23 mg;Mg:20 g;Cr:60 mg;Mo: 500 mg; Zn: 6000 mg; Fe: 3000 mg; l: 80 mg.

A composição química média da ração total misturada fornecida aos cordeiros (Quadro 2) foi obtida com amostragens diárias e formação de amostras compostas semanais, que foram analisadas no Laboratório de Nutrição Animal da UFPR. Os níveis de fibra em detergente ácido e fibra em detergente neutro foram determinados de acordo com a metodologia descrita por Van Soest et al. (1991), enquanto os demais nutrientes foram obtidos segundo Silva e Queiroz (2002). A dieta atingiu o valor mínimo de 2,4 Mcal EM/kg de MS, estipulado para não limitar a ingestão em testes de consumo em bovinos, conforme a Beef Improvement Federation (BIF, 2010).
Quadro 2 - Composição bromatológica da dieta experimental

\begin{tabular}{lc}
\hline \multicolumn{1}{c}{ Composição química } & \% (MS) \\
\hline Matéria seca & 86,71 \\
Proteína bruta & 13,52 \\
Extrato etéreo & 2,77 \\
Resíduo mineral & 6,26 \\
Fibra em detergente ácido & 21,12 \\
Fibra em detergente neutro & 39,43 \\
Nutrientes digestíveis totais & 65,09 \\
Cálcio & 1,04 \\
Fósforo & 0,42 \\
\hline
\end{tabular}

Fonte: Dados da Pesquisa.

As sobras foram pesadas e amostradas diariamente, permitindo-se a formação de amostras compostas semanais. Os teores de MS da ração ofertada e das sobras foram determinados semanalmente para obtenção do CMSobs, que foi calculado pela média da subtração da quantidade de alimento diário fornecido pela quantidade de sobras diárias (ambos ajustados para MS).

Durante o período experimental, foram realizadas cinco pesagens (em condição de jejum de 12 horas) para determinação do ganho de peso médio diário (GPD), calculado por regressão linear entre o tempo de experimento e os PV individuais. Ao término do experimento, os animais apresentavam em média 67,9 $\pm 6,2$ de PV final (PVF). 0 consumo de MS estimado ou predito (CMSest) foi modelado com auxílio do programa estatístico R Development Core Team (2010), por regressão do CMSobs em função do peso metabólico médio (PM $=$ PVmédio ${ }^{0,75}$ ) e do GPD de cada animal durante o período, conforme a equação e seu respectivo coeficiente de determinação: CMSest $=0,66170+1,97464 * \mathrm{GPD}$ $+0,02258 *$ PM $\left(R^{2}=0,64\right)$. Utilizando a metodologia proposta por Koch et al. (1963), o CAR de cada animal foi obtido da diferença entre o CMSobs e o CMSest. A CA foi calculada como a relação direta do CMSobs diário pelo GPD, enquanto seu inverso representou a EA.

No início e no fim do experimento (dia 0 após a adaptação e dia 70 do período experimental, respectivamente) foram feitas avaliações de carcaça por meio de imagens de ultrassom (ALOKA PROSOUND $2^{\circledR}$ - transdutor linear multifrequencial de $6 \mathrm{MHz}$ ) com determinação das seguintes características: área de olho de lombo (AOL), profundidade do músculo Longissimus e espessura de gordura subcutânea 
(EGS) entre a $12^{\mathrm{a}}$ e $13^{\mathrm{a}}$ costelas. Também foram avaliados os ganhos dessas características no decorrer do tempo, subtraindo-se os valores da primeira avaliação dos valores da segunda avaliação.

Para efeito de comparação, estabeleceram-se duas classes de CAR entre os animais com base no desvio padrão (DP), os menos eficientes $\left(0,5^{*} \mathrm{DP}\right.$ acima da média, $n=4)$ e os mais eficientes $\left(0,5^{*}\right.$ DP abaixo da média, $n=5$ ). Estimaram-se as correlações simples de Pearson entre CAR e as demais variáveis com o procedimento analítico cor.test do programa estatístico R Development Core Team (2010) e, nesse mesmo programa, os dados das distintas classes de eficiência foram submetidos à análise de variância para verificar as diferenças pelo teste $\mathrm{F}$ a 5\% de significância.

\section{Resultados}

0 consumo médio de ração $(\mathrm{n}=13)$ durante o período experimental foi de 1,688 $\pm 0,160 \mathrm{~kg}$ MS/dia, o que correspondeu a aproximadamente 2,90 \pm 0,23\% do PV, valor mínimo de CAR foi de -0,076 kg MS/dia, enquanto que o máximo foi de $0,014 \mathrm{~kg}$ MS/dia, resultando em uma diferença na ingestão de 0,090 kg de MS/dia entre o animal mais eficiente e o menos eficiente, confirmando a existência de variação considerável no consumo entre indivíduos, o que pode também ser constatado pelo desvio padrão do CAR que foi de $0,096 \mathrm{~kg}$ de MS/dia.

Como pode ser verificado no Quadro 3, o CAR se mostrou correlacionado ( $p<0,05)$ com o consumo tanto em kg de MS/dia quanto em percentual do PV.

Como já era esperado, as classes diferiram no CAR e os cordeiros de CAR baixo tiveram menor consumo de MS em percentual do PV $(p=0,01)$ e tendência a apresentar menor consumo em kg MS/dia $(p=0,08)$. Não foram observadas diferenças quanto ao GPD, PVI, PVF e PM entre as classes de CAR ( $p>0,05)$, corroborando a capacidade deste na identificação de animais mais eficientes, com ganhos similares e menor ingestão de alimento. Esse fato pode ser observado também nas diferenças detectadas na CA e na EA, ou seja, a classe mais eficiente pelo CAR apresentou também melhor eficiência quando avaliada pela CA e pela EA.

A CA apresentou correlação forte e negativa $(r=-0,99)$ com a EA, o que já era esperado, pois, nesse caso o cálculo de uma variável leva em consideração o inverso da outra. 0 coeficiente de correlação entre a CA e o CAR foi de $+0,51(p=0,07)$ e, diferentemente deste último, a CA se mostrou fortemente correlacionada $(\mathrm{p}<0,05)$ com o GPD e moderadamente com o PVF dos animais no experimento.

Quadro 3 - Coeficientes de correlação de Pearson $(n=13)$ entre as medidas de desempenho e eficiência e o consumo alimentar residual (CAR) e conversão alimentar (CA); e médias \pm desvios padrão obtidos para essas características em cordeiros com alto ou baixo CAR

\begin{tabular}{|c|c|c|c|c|c|}
\hline \multirow{2}{*}{ Variáveis } & \multicolumn{2}{|c|}{ Correlações } & \multicolumn{2}{|c|}{ CAR (classes) } & \multirow{2}{*}{ p } \\
\hline & $r_{\text {CAR }}$ & $r_{C A}$ & Alto $(n=4)$ & Baixo $(n=5)$ & \\
\hline $\mathrm{CMS}_{\mathrm{obs}}$ & $0,60^{* *}$ & $-0,33$ & $1,740 \pm 0,107$ & $1,570 \pm 0,135$ & 0,08 \\
\hline CMS (\%PV) & $0,71^{* * *}$ & 0,06 & $3,00 \pm 0,15 a$ & $2,63 \pm 0,10 b$ & 0,01 \\
\hline GPD (kg/dia) & 0,00 & $-0,83^{* * *}$ & $0,253 \pm 0,027$ & $0,266 \pm 0,040$ & 0,59 \\
\hline PVI & 0,03 & $-0,14$ & $49,813 \pm 4,643$ & $50,650 \pm 5,246$ & 0,81 \\
\hline PVF & $-0,03$ & $-0,61^{* *}$ & $66,125 \pm 4,404$ & $68,650 \pm 7,815$ & 0,58 \\
\hline PM & 0,00 & $-0,43$ & $20,998 \pm 1,202$ & $21,444 \pm 1,752$ & 0,68 \\
\hline CA & $0,51^{*}$ & - & $6,915 \pm 0,530 a$ & $5,944 \pm 0,382 b$ & 0,01 \\
\hline EA & $-0,44$ & $-0,99^{* * *}$ & $0,145 \pm 0,01 \mathrm{~b}$ & $0,169 \pm 0,01 \mathrm{a}$ & 0,02 \\
\hline CAR & - & $0,51^{*}$ & $0,105 \pm 0,054 a$ & $-0,101 \pm 0,034 b$ & 0,00 \\
\hline
\end{tabular}

Lagenda: CMSobs = Consumo de MS observado (kg de MS/dia); GPD = ganho de peso médio diário; PVI = peso vivo inicial; PVF = peso vivo final; PM = peso metabólico médio; $C A=$ conversão alimentar; $E A=$ eficiência alimentar; ${ }^{*}=p<0,10 ;{ }^{* *}=p<0,05 ;{ }^{* * *}=p<0,01$.

Fonte: Dados da pesquisa.

Nota: Letras distintas na mesma linha diferem pelo teste $F(p<0,05)$.

Rev. Acad., Ciênc. Agrár. Ambient., Curitiba, v. 10, n. 2, p. 129-135, abr./jun. 2012 
O CAR não se apresentou correlacionado $(p>0,05)$ com nenhuma das características de carcaça avaliadas neste estudo e também não houve diferença $(p>0,05)$ entre as classes de eficiência alimentar pelo CAR para essas variáveis analisadas por ultrassonografia (Quadro 4).

Entretanto, ressalta-se a correlação moderada e positiva $(r=+0,52)$ estimada entre oCAR e oganho em $\operatorname{EGS}(p=0,07)$ ao longo do experimento. Apesar disso, a correlação não foi suficiente para mostrar diferenças significativas entre as classes de CAR ( $p>0,05)$, mesmo os valores absolutos assim indicando.

\section{Discussão}

A importância do CAR na identificação e seleção de animais de produção mais eficientes tem sido destacada por diversos autores. De acordo com Gomes et al. (2012), o CAR pode ser considerado um critério interessante e efetivo para comparação de dados entre grupos contemporâneos. Dentre os pontos favoráveis à utilização dessa medida de eficiência na produção animal, com base nos resultados com bovinos, destaca-se o fato da ausência de relações com medidas de crescimento, tais como o PV e a taxa de ganho do animal (ARTHUR et al., 2001; HERD et al., 2003; SCHENKEL et al., 2004), fato também confirmado no estudo com ovinos.
Em contrapartida, seria importante verificar a existência de correlações genéticas, assim como as correlações fenotípicas estimadas neste trabalho entre CA e GPD e PVF para ovinos. Para bovinos, os prejuízos da utilização da CA como critério de seleção são frequentemente discutidos, graças às relações desta com características de crescimento e tamanho corporal, que levariam à obtenção de animais de maior porte e, consequentemente, com maiores exigências nutricionais de mantença (ARTHUR; HERD, 2008).

Para bovinos da raça Nelore, Castilhos et al. (2010) estimaram correlações de mesmo sentido similares às deste experimento entre CAR e CMS $(\mathrm{r}=+0,58)$, entre CAR e CMS\%PV $(\mathrm{r}=+0,72)$ e entre CAR e CA $(r=+0,42)$; e Santana et al. (2012) estimaram coeficientes de +067 , $+0,82$ e $+0,28$, entre essas mesmas características, respectivamente. Da mesma forma, porém em pesquisa com ovinos, Knott et al. (2010) descreveram correlações semelhantes de $+0,68$ entre CAR e CMS, e +0,73 entre CAR e CA, enquanto Redden et al. (2010) estimaram correlações de $+0,77$ e $+0,47$, respectivamente entre tais variáveis. Paula et al (2013) em experimento com cordeiros da raça Ile de France, encontrou coeficientes de correlação de $+0,81$ entre CAR e CMS, $+0,90$ entre CAR e CMS\%PV, e de $+0,63$ entre CAR e CA. Destaca-se que ainda há carência de

Quadro 4 - Coeficientes de correlação de Pearson entre medidas da carcaça avaliadas por ultrassom e o consumo alimentar residual $\left(\mathrm{r}_{\mathrm{CAR}}\right)$, e médias e desvios padrão obtidos para essas características em ovinos com alto ou baixo CAR

\begin{tabular}{lcccc}
\hline \multirow{2}{*}{ Variáveis } & Correlações & \multicolumn{2}{c}{ CAR (classes) } & \\
\cline { 2 - 3 } & $\mathbf{r}_{\text {CAR }}$ & Alto $(\mathbf{n}=\mathbf{4})$ & Baixo $(\mathbf{n}=\mathbf{5})$ & \\
\hline AOL inicial $\left(\mathrm{cm}^{2}\right)$ & 0,04 & $11,87 \pm 1,89$ & $12,18 \pm 1,23$ & 0,77 \\
AOL final $\left(\mathrm{cm}^{2}\right)$ & 0,11 & $17,15 \pm 2,53$ & $17,57 \pm 0,87$ & 0,73 \\
AOL ganho $\left(\mathrm{cm}^{2}\right)$ & 0,08 & $5,28 \pm 0,93$ & $5,38 \pm 1,07$ & 0,88 \\
EGS inicial $\left(\mathrm{cm}^{2}\right)$ & $-0,18$ & $0,43 \pm 0,05$ & $0,46 \pm 0,17$ & 0,70 \\
EGS final $\left(\mathrm{cm}^{2}\right)$ & 0,30 & $0,78 \pm 0,26$ & $0,65 \pm 0,16$ & 0,47 \\
EGS ganho $\left(\mathrm{cm}^{2}\right)$ & $0,52^{*}$ & $0,34 \pm 0,22$ & $0,19 \pm 0,07$ & 0,20 \\
Profundidade inicial $(\mathrm{cm})$ & $-0,03$ & $2,33 \pm 0,36$ & $2,44 \pm 0,23$ & 0,58 \\
Profundidade final $(\mathrm{cm})$ & 0,02 & $3,14 \pm 0,45$ & $3,27 \pm 0,18$ & 0,56 \\
Profundidade ganho $(\mathrm{cm})$ & 0,06 & $0,81 \pm 0,14$ & $0,83 \pm 0,15$ & 0,86 \\
\hline
\end{tabular}

Legenda: $\mathrm{AOL}=$ Área de olho de lombo; $\mathrm{EGS}=$ Espessura de gordura subcutânea; Profundidade = profundidade do músculo Longissimus; ${ }^{*}=p<0,10$. Fonte: Dados da pesquisa. 
estudos sobre CAR na espécie ovina, o que dificulta a discussão dos resultados. Porém, de modo geral, os resultados disponíveis na literatura concordam com os descritos neste trabalho.

Os sentidos e as dimensões das correlações estimadas enfatizam o potencial do CAR como alternativa para mensurar a eficiência alimentar e corroboram sua independência dos aspectos de tamanho corporal e velocidade de crescimento, tais como GPD, PVI, PVF e PM, anuindo com o proposto inicialmente para bovinos por Koch et al. (1963).

A amplitude de variação e o desvio padrão do CAR neste trabalho se mostraram inferiores aos encontrados por Paula et al. (2012), em que o cordeiro mais eficiente apresentou CAR de -0,426 kg MS/dia e o menos eficiente de 0,149 kg MS/dia, e desvio padrão de 0,129 kg MS/dia. Provavelmente, essas divergências ocorreram pela menor idade e pelas diferenças inerentes à raça (Ile de France) dos animais experimentais utilizados por esses autores.

Em relação às características de carcaça, Knott et al. (2003), em trabalho com ovinos, encontraram correlação negativa entre carcaça magra e CAR, indicando que, quanto menor o CAR, mais magra seria a carcaça dos animais. Para bovinos de corte da raça Nelore, Leme e Gomes (2007) descreveram que animais mais eficientes (CAR baixo) tiveram maiores AOL e também menor EGS. Esses relatos sugerem que animais mais eficientes pelo CAR tendem a apresentar menos gordura nas carcaças, podendo prejudicar a qualidade e acabamento. Contudo, de acordo com Santana et al. (2012), para bovinos e com os resultados deste estudo para ovinos, não houve correlações significativas para as características de carcaça em questão, e os animais mais eficientes pelo CAR não apresentaram condições inferiores no que diz respeito à deposição de gordura na carcaça, apresentando portanto, acabamento similar aos animais menos eficientes.

A ausência de resultados significativos pode ser justificada pelo fato de este trabalho ter sido conduzido com animais jovens e a deposição de gordura não ser tão efetiva para ovinos em crescimento. Em concordância com essa hipótese, a tendência à significância apresentada no ganho em EGS, está indicando que os cordeiros de menor CAR podem depositar menos gordura no decorrer do tempo. Dessa forma, sugere-se a realização de pesquisas complementares com ovinos de idade e maturidade mais avançadas e também por um período mais longo, para confirmar os resultados encontrados em relação ao ganho em EGS.

\section{Conclusão}

O CAR se mostrou útil para a classificação da eficiência alimentar de ovinos e, no estágio de maturidade fisiológica em que os ovinos foram avaliados, não houve relações com medidas de tamanho corporal, não ocasionando prejuízos ao desempenho e sem interferência nas características de carcaça destes animais. Assim, a seleção pelo CAR em ovinos parece resultar em animais de desempenho similar sem afetar a qualidade e acabamento das carcaças.

\section{Referências}

ALMEIDA, R. et al. Consumo alimentar residual: um novo parâmetro para avaliar a eficiência alimentar de bovinos de corte. In: REUNIÃO ANUAL DA SOCIEDADE BRASILEIRA DE ZOOTECNIA, 41, 2004, Campo Grande. Anais... Campo Grande: Sociedade Brasileira de Zootecnia, 2004. p. 4.

ARTHUR, P. F. et al. Genetic and phenotypic variance and covariance components for feed intake, feed efficiency, and other postweaning traits in Angus cattle. Journal of Animal Science, v. 79, p. 2805-2811, 2001.

ARTHUR, P. F.; HERD, R. M. Residual feed intake in beef cattle. Revista Brasileira de Zootecnia, v. 37, p. 269279, 2008.

BEEF IMPROVEMENT FEDERATION - BIF. Guidelines for uniform beef improvement programs. Raleigh: U.S. Department of Agriculture, North Carolina State University, 2010.

CASTILHOS, A. M. et al. Feed efficiency of Nellore cattle selected for postweaning weight. Revista Brasileira de Zootecnia, v. 39, p. 2486-2493, 2010.

GOMES, R. C. et al. Ingestão de alimentos e eficiência alimentar de bovinos e ovinos de corte. Ribeirão Preto: Fundação de Pesquisas Científicas de Ribeirão Preto (FUNPEC, RP), 2012.

HERD, R. M. et al. Reducing the cost of beef production through genetic improvement in residual feed intake: opportunity and challenges to application. Journal of Animal Science, v. 81, p. 9-17, 2003. 
LANNA, D. P. D; ALMEIDA, R. Residual feed intake: um novo critério de seleção? In: SIMPÓSIO DA SOCIEDADE BRASILEIRA DE MELHORAMENTO ANIMAL, 5, 2004, Pirassununga. Anais... Pirassununga: Sociedade Brasileira de Melhoramento Animal, 2004. p. 12.

LEME, P. R.; GOMES, R. C. Características de carcaça de novilhos Nelore com diferente consumo alimentar residual. In: REUNIÓN ASOCIASÓN LATINOAMERICANA DE PRODUCION ANIMAL, 20, 2007, Cuzco. Anais... Cuzco: ALPA, 2007.

KNOTT, S. A. et al. Feed efficiency and body composition are related to cortisol response to adrenocorticotropin hormone and insulin-induced hypoglycemia in rams. Domestic Animal Endocrinology, v. 39, p. 137 146, 2010.

KNOTT, S. A. et al. Relationship between body composition, net feed intake and gross feed conversion efficiency in composite sire line sheep. EAAP Publication, 109, 2003, p. 525-528.

KOCH, R. M. et al. Efficiency of feed use in beef cattle. Journal of Animal Science, v. 22, p. 486-494, 1963.

PAULA, E. F. E. et al. Consumo alimentar residual e sua relação com medidas de desempenho e eficiência e características in vivo da carcaça de cordeiros. Arquivo Brasileiro de Medicina Veterinária e Zootecnia, v.65, n.2, p.566-572, 2013.

PAULA, E. F. E. et al. Características de carcaça obtidas por ultrassonografia e o consumo alimentar residual de cordeiros da raça Ile de France. Revista Synergismus scyentifica UTFPR, v. 7, n. 1, 2012.

R DEVELOPMENT CORE TEAM. R: A language and environment for statistical computing . R Foundation for Statistical Computing, Vienna, Austria, 2010. Disponível em: <http://www.R-pro-ject.org>.
REDDEN, R. et al. Growth rate alters residual feed intake and feeding behavior in yearling ewes. In: U.S SHEEP RESEARCH AND OUTREACH PROGRAMS, 2010 Tennessee. Proceedings... Tennessee, 2010, p. 26-28.

REDDEN, R. R. et al. Residual feed efficiency established in a post-weaning growth test may not result in more efficient ewes on the range. Small Ruminant Research, v. 96, p. 155-159, 2011.

SANTANA, M. H. A. et al. Feed efficiency and its correlations with carcass traits measured by ultrasound in Nellore bulls. Livestock Science, v. 145, p. 252-257, 2012.

SCHENKEL, F. S. et al. Genetic parameters and breed differences for feed efficiency, growth, and body composition traits of young beef bulls. Canadian Journal of Animal Science, v. 84, p. 177-185, 2004.

SILVA, D. J.; QUEIROZ, A. C. Análise de alimentos: métodos químicos e biológicos. Viçosa: Universidade Federal de Viçosa, 2002.

VAN SOEST, P. J. et al. Methods for dietary fiber, neutral detergent fiber, and no starch polysaccharides in relation to animal nutrition. Journal of Dairy Science, v. 74 , n. 10 , p. 3583-3597, 1991

Recebido: 15/08/2012

Received: 08/15/2012

Aprovado: 23/10/2012

Approved: 10/23/2012 